University of New Hampshire

University of New Hampshire Scholars' Repository

Space Science Center

Institute for the Study of Earth, Oceans, and

Space (EOS)

3-10-2003

\title{
Nonsolar astronomy with the Reuven Ramaty High Energy Solar Spectroscopic Imager (RHESSI)
}

\author{
David M. Smith \\ University of California - Berkeley \\ Robert P. Lin \\ University of California - Berkeley \\ K Hurley \\ University of California - Berkeley \\ Wayne Coburn \\ University of California - Berkeley \\ G J. Hurford \\ University of California - Berkeley
}

See next page for additional authors

Follow this and additional works at: https://scholars.unh.edu/ssc

Part of the Astrophysics and Astronomy Commons

\section{Recommended Citation \\ David M. Smith ; Robert P. Lin ; Kevin C. Hurley ; Wayne Coburn ; Gordon J. Hurford ; Claudia Wigger ; W. Hajdas ; Alex Zehnder and Mark L. McConnell "Nonsolar astronomy with the Reuven Ramaty High Energy Solar Spectroscopic Imager (RHESSI)", Proc. SPIE 4851, X-Ray and Gamma-Ray Telescopes and Instruments for Astronomy, 1163 (March 10, 2003); doi:10.1117/12.461415; http://dx.doi.org/10.1117/} 12.461415

This Conference Proceeding is brought to you for free and open access by the Institute for the Study of Earth, Oceans, and Space (EOS) at University of New Hampshire Scholars' Repository. It has been accepted for inclusion in Space Science Center by an authorized administrator of University of New Hampshire Scholars' Repository. For more information, please contact Scholarly.Communication@unh.edu. 


\section{Authors}

David M. Smith, Robert P. Lin, K Hurley, Wayne Coburn, G J. Hurford, Claudia Wigger, W Hajdas, Alex Zehnder, and Mark L. McConnell 


\title{
Non-solar astronomy with the Reuven Ramaty High Energy Solar Spectroscopic Imager (RHESSI)
}

\author{
David M. Smith ${ }^{a}$, Robert P. Lin ${ }^{a, b}$, Kevin Hurley ${ }^{a}$, Wayne Coburn ${ }^{a}$, Gordon Hurford ${ }^{a}$, \\ Claudia Wigger ${ }^{c}$, Wojtek Hajdas ${ }^{c}$, Alex Zehnder ${ }^{c}$, \\ Mark McConnell ${ }^{d}$, \\ and the RHESSI team \\ ${ }^{a}$ Space Sciences Laboratory, University of California, Berkeley, CA, USA \\ ${ }^{b}$ Department of Physics, University of California, Berkeley, CA, USA \\ ${ }^{c}$ Paul Scherrer Institut, Villigen, Switzerland \\ ${ }^{d}$ Space Science Center, University of New Hampshire, Durham, NH
}

\begin{abstract}
The Reuven Ramaty High Energy Solar Spectroscopic Imager (RHESSI) is a NASA Small Explorer satellite designed to study hard x-ray and gamma-ray emission from solar flares. In addition, its high-resolution array of germanium detectors can see photons from high-energy sources throughout the Universe. Here we discuss the various algorithms necessary to extract spectra, lightcurves, and other information about cosmic gamma-ray bursts, pulsars, and other astrophysical phenomena using an unpointed, spinning array of detectors. We show some preliminary results and discuss our plans for future analyses. All RHESSI data are public, and scientists interested in participating should contact the principal author.
\end{abstract}

Keywords: X-rays, gamma-rays, astronomy, astrophysics, germanium, detectors

\section{INTRODUCTION}

The Reuven Ramaty High Energy Solar Spectroscopic Imager (RHESSI) was launched on February 5, 2002 into low-Earth orbit. It is a NASA Small Explorer mission built at the University of California, Berkeley (Robert P. Lin, Principal Investigator), the NASA Goddard Space Flight Center, the Paul Scherrer Institut in Switzerland, and Spectrum Astro, Inc., with participation by a number of other institutions.

RHESSI's primary science goals are imaging spectroscopy and high-resolution nuclear spectroscopy of solar flares. The instrument consists of 9 large, coaxial germanium detectors (cooled to $75 \mathrm{~K}$ by a mechanical cooler) that cover an energy range of $3 \mathrm{keV}$ to $17 \mathrm{MeV}$. Each detector sits immediately below a fine grid of tungsten or molybdenum slats, with an identical grid $1.5 \mathrm{~m}$ above. The grid pairs make up a Rotation Modulation Collimator (RMC) system for high-resolution imaging. As the spacecraft rotates at $15 \mathrm{rpm}$, each grid pair modulates the count rate in the detector below. Each grid pair has a different spacing and therefore covers a different angular scale. The temporal modulations can be deconvolved to produce an image of the field of view of the detectors through the grid pairs, which is about $1.5^{\circ}$ in radius. The finest grid images down to 2.3 arcsec.

Each germanium detector is a closed-end coaxial cylinder with a volume of over $300 \mathrm{~cm}^{3}$, electronically segmented into a thin front segment and a thick rear segment. The $\sim-4000 \mathrm{~V}$ bias is applied on the outer (n-type) contact, and the inner (p-type) contact is divided in two pieces separated by an insulating step; signals are read out separately from each part. The rear segments view nearly half the sky through side walls of only $4 \mathrm{~mm}$ of aluminum, which have significant transmission above $25 \mathrm{keV}$. The front segments shield the rear segments from solar photons below $100 \mathrm{keV}$ and view the Sun through beryllium windows and a small amount of insulating blanket, giving them a useful energy range down to about $3 \mathrm{keV}$. Above about $100 \mathrm{keV}$, the detectors have a significant response to photons from any direction in the sky.

\footnotetext{
Send correspondence to D. M. S. at dsmith@ssl.berkeley.edu
} 

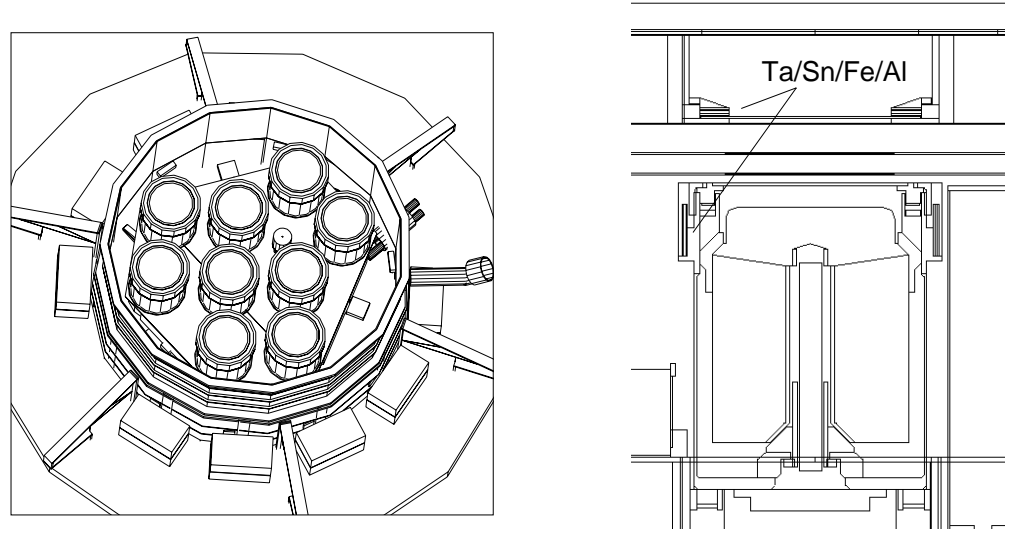

Figure 1. Left: arrangement of the RHESSI detectors in the cryostat. Right: cross section of one detector showing segmentation and the location of graded $-\mathrm{Z}(\mathrm{Ta} / \mathrm{Sn} / \mathrm{Fe} / \mathrm{Al})$ shielding.

Fig. 1 (left) shows the arrangement of the detectors in the cryostat and Fig. 1 (right) shows a cross-section of one of the detectors. The boundary field line separating the front and rear segments is shown; at the inner bore it ends at the step that interrupts the contact. Also shown in Fig. 1 (right) are rings of graded-Z material (from outside to inside, $\mathrm{Ta} / \mathrm{Sn} / \mathrm{Fe} / \mathrm{Al}$ ) that shield the rear segments from soft solar photons (horizontal ring at top) and the front segments from background photons (vertical ring to the side).

The energy resolution of the front segments is about $1 \mathrm{keV}$ full width at half maximum (FWHM) below 100 $\mathrm{keV}$. They are read out from $3 \mathrm{keV}$ to $2.7 \mathrm{MeV}$. Only photons from the solar field of view can come in at low energies. The rear segments, which are used for most non-solar observations, are read out from $20 \mathrm{keV}$ to 17 $\mathrm{MeV}$ and have an energy resolution of about $4 \mathrm{keV}$ FWHM at $2 \mathrm{MeV}$ when summed together.

RHESSI data are telemetered one photon at a time with full energy information (bins $1 / 3 \mathrm{keV}$ wide below $2.7 \mathrm{MeV}$ and $2.1 \mathrm{keV}$ wide above) and full time resolution (in units of binary microseconds). Absolute time calibration is known to a few milliseconds. To conserve the onboard memory and downlink capacity, events are turned off while the spacecraft is going through the South Atlantic Anomaly, and the front segments are turned off during spacecraft night. On some occasions, also to save memory, events in the rear segments below a threshold energy (usually about $400 \mathrm{keV}$ ) are "decimated" so that only 1 out of $\mathrm{N}$ are actually collected, where $\mathrm{N}$ is a small integer chosen by command.

Because the data that come to the ground all have full energy and time resolution, a variety of analyses are possible on the same dataset without the restrictions usually encountered by having to put an instrument into "burst mode", "pulsar mode", etc. The possible non-solar observations fall into four categories based on the technique used to distinguish between source and background counts: observations of burst emission, imaged observations, observations of pulsed emission, and observations using occultation of the source (either by the Earth or by parts of the spacecraft, including the detectors, as it rotates).

\section{BURSTS}

Fast events such as cosmic gamma-ray bursts (GRBs) and bursts from Soft Gamma Repeaters (SGRs), which last milliseconds to tens of seconds, are among the easiest cosmic sources to analyze with an uncollimated instrument. Background is removed by simply subtracting the spectrum just before and after the event; spin modulation aside, background does not vary significantly on timescales less than a few minutes. 
GRB020715, 19:21:03 - 19:21:13 UTC
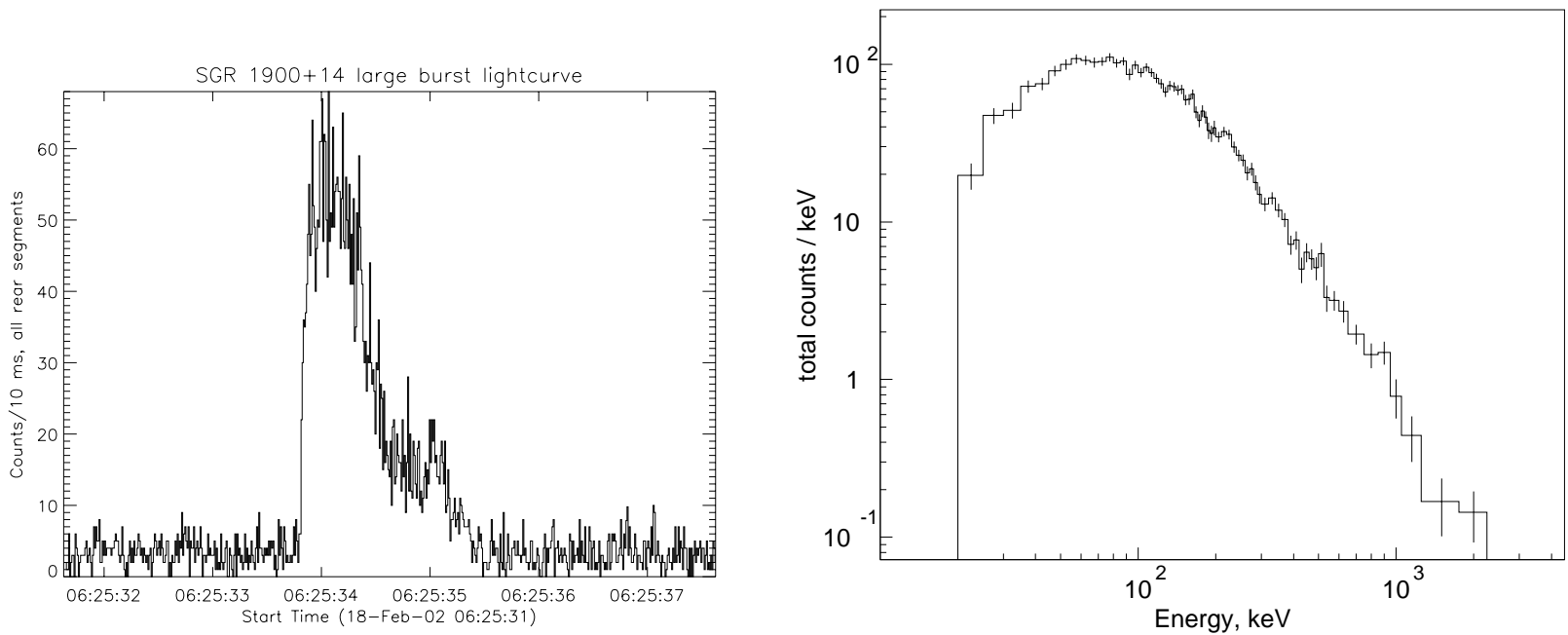

Figure 2. Left: lightcurve of the bright burst from SGR $1900+14$ on February 18, 2002. For the second or two it was active, it was far brighter than the rest of the sky, the Earth, and the instrumental background combined. Right: energy spectrum (background-subtracted, but not corrected for instrument response) from GRB 020715.

Fig. 2 (left) shows the lightcurve of emission from the extremely bright burst of SGR 1900+14 observed by RHESSI on February 18, 2002, less than a week after its detectors had been turned on. This object is thought to be a magnetar, an exotic neutron star in our Galaxy with a magnetic field several orders of magnitude higher than "ordinary" pulsars, its outbursts being the result of "starquakes" caused by the release of crustal tension due to changes in the magnetic field. ${ }^{1}$

Fig. 2 (right) shows the energy spectrum (background-subtracted) from a bright GRB. The SGR spectrum is much softer, disappearing above about $150 \mathrm{keV}$. Unlike the relatively local SGR events, GRBs are now commonly believed to be among the most distant phenomena known, and are thought to be caused by massive explosions in distant galaxies. The leading candidates for their sources are more-than-usually-bright supernovae ("hypernovae" $)^{2}$ and the final merger of binary systems composed of two neutron stars. ${ }^{3}$ RHESSI is the largest high-resolution instrument ever to observe GRBs, and with its large field of view it gives us the best chance of any instrument past, current, or planned for finding narrow emission or absorption lines in GRB and SGR spectra. Over a dozen GRB have been observed by RHESSI so far, and a public database of them is currently being maintained on the World Wide Web at http://grb.web.psi.ch/.

In addition, RHESSI is being integrated into the Interplanetary Network (IPN) of GRB detectors. ${ }^{4}$ The IPN consists of gamma-ray detectors on diverse missions throughout the solar system. When a GRB occurs, its lightcurves from each instrument are cross-correlated to find the relative arrival times of the wavefront at each spacecraft. Depending on the burst position, its brightness, and the number of spacecraft observing, error boxes can range from a few to a few hundred square arcminutes. RHESSI lightcurves have already been used to help create or to refine several IPN error boxes. Speed is critical since these error boxes are used to trigger searches for rapidly fading x-ray and optical transients associated with the burst. RHESSI data are stored in a large solid-state memory on board and telemetered during passes over the Berkeley Ground Station and other stations. The delay is about 12-72 hours, which is often fast enough to be useful for the IPN. Software is under development to automatically search RHESSI data for bursts and then email lightcurves tailored for the IPN cross-correlations.

Finally, RHESSI can also provide a crude position measurement for a burst by itself. As the satellite rotates 
at $15 \mathrm{rpm}$, the nine germanium detectors occult each other, so that a GRB coming in from the side is modulated. The azimuth angle can be obtained from the phase of the modulation. For a burst of $<<4 \mathrm{~s}$, simple count ratios in the different rear segments can be used and compared to models for different azimuthal angles. The polar angle can be obtained from the following three considerations:

1. For a GRB coming from the side ( $90^{\circ}$ from the spacecraft axis), the amplitude of the modulation is maximal, while for a GRB coming from the back or front direction, the signal shows no modulation at all.

2. Similarly, a GRB near $90^{\circ}$ gives more counts in the outer six detectors and fewer in the inner three, whereas for a burst near the axis (front or back) the count rates will be nearly equal.

3. The ambiguity between bursts from the front and behind can be resolved by looking at the the ratio between the total counts in the front and rear segments of the detectors.

Preliminary results and simulations indicate that we can find positions to an angular uncertainty of about $10^{\circ}$ in polar angle and $15^{\circ}$ in azimuthal angle by comparing the observed data to simulations of bursts from different directions using these criteria.

\section{IMAGING NON-SOLAR SOURCES}

Two bright Galactic sources drift into the solar field of view of RHESSI's RMC imaging system as the spacecraft follows the Sun across the sky: the Crab nebula and pulsar (ecliptic latitude $-1.3^{\circ}$ ), and the "Z" source (low mass x-ray binary) GX $5-1$ (ecliptic latitude $-1.6^{\circ}$ ). Modulation by the imager separates source and background during these periods, resulting in very clean high-resolution spectra. More importantly, the Crab nebula is by far the brightest extended hard x-ray source in the sky, and of a perfect size to be imaged with the RMCs. Only one image above about $15 \mathrm{keV}$ has ever been produced, ${ }^{5}$ by a balloon payload with a resolution of about 15 arcsec. Soft x-ray images have been made with ROSAT, ${ }^{6}$ Chandra, ${ }^{7}$ and XMM-Newton. ${ }^{8}$ The Chandra and Newton images show the nebula shrinking with increasing energy and the structures (torus and jets) increasing in contrast. The Crab radio wisps near the pulsar are known to evolve rapidly, so it will be interesting to compare hard x-ray images of that region from one year to the next.

RHESSI was designed only to point at the Sun, but algorithms are currently under development for offset pointing, with offset capability up to 10 degrees likely and up to 25 degrees possible. After the Sun had passed its closest approach to the Crab in June 2002 we attempted to point back directly to the Crab, but this first attempt was not successful. An improved algorithm will be tested on GX 5-1 in December 2002 to prepare us for the next Crab encounter. With the improved counting statistics achieved by both direct pointing and longer exposure, we should be able to image the nebula up to $100 \mathrm{keV}$ or higher. The ultimately attainable angular resolution in off-pointing mode will be limited by the performance of the aspect sensors rather than the 2.3 arcsec scale of the finest grid, but it should still be significantly better than the 15 arcsec already achieved. ${ }^{5}$ Other off-pointing candidates are likely to be exceptionally bright transient sources (pulsars, black-hole x-ray novae, nearby classical novae, etc.). Most will not be extended enough to image (although x-ray jets are possible in a bright transient binary), but the implicit background-subtraction of the imaging process will allow excellent spectroscopy of these systems, and RHESSI's spatial resolution can provide localizations of new sources for counterpart searches.

\section{PULSAR STUDIES}

The pulsed emission from solitary and accreting pulsars can be separated from background by simply "folding" the lightcurve in each energy band at the pulsar period, accumulating the data in a number of different phase bins. Although the RHESSI background varies strongly with time at all energies, none of these variations will be at the same frequency as the pulsar under consideration, and therefore they will average out to a constant DC background level. For fast pulsars or for very long data accumulations, the photon arrival times must be corrected to the solar system barycenter before each photon is placed into a phase bin. Fig. 3 shows a folded lightcurve for the Crab pulsar over a broad energy range $(12-38 \mathrm{keV})$ using approximately 1 day of data taken 


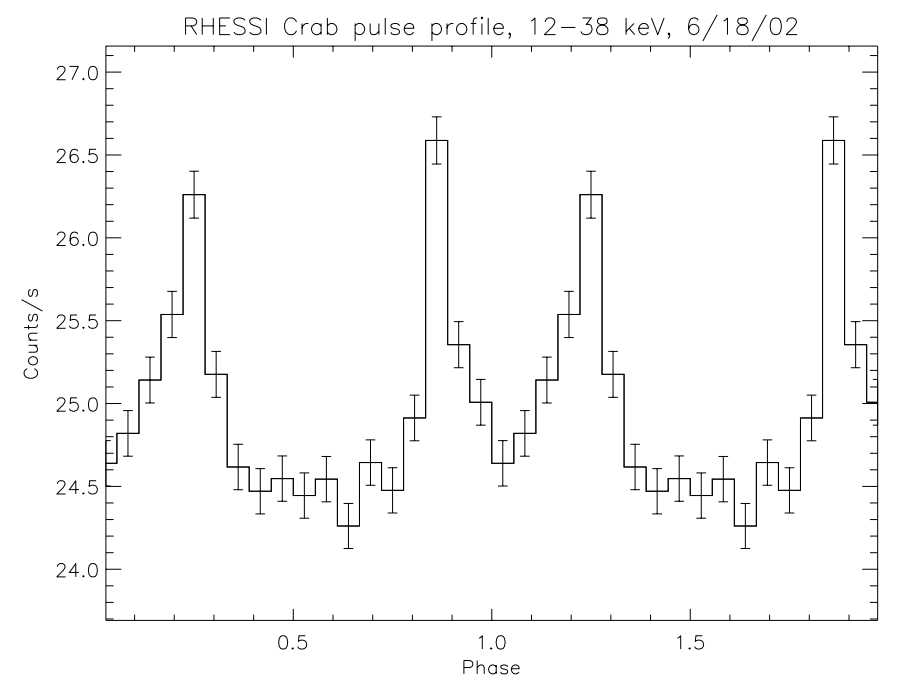

Figure 3. RHESSI pulse profile for the Crab pulsar using front segment data in June 2002. The data are repeated over two cycles to more clearly show the shape.

in the front segments while the Crab passed through the edge of the solar field of view in June 2002. Other pulsars will require much longer accumulations, both because they are fainter and because they will generally have to be analyzed using the rear segments, which have a higher background.

The hard x-ray spectra of some accreting pulsars contain features that arise due to resonant scattering of photons by electrons whose energies are quantized perpendicular to the magnetic field. These line-like features, known as cyclotron lines, are the only direct measure of neutron star magnetic fields. First discovered ${ }^{9}$ in Her $\mathrm{X}-1$, such structures have now been seen in 13 neutron star sources and give magnetic fields in the range $(1-5) \times 10^{12}$. Coburn et al. ${ }^{10}$ give a comprehensive review.

Recent simulations ${ }^{11,12}$ find that the detailed line profiles of cyclotron lines are sensitive probes of not only the physical parameters in the region (e.g. the magnetic field and electron temperature), but also the geometric properties of the polar region (e.g. the emission geometry and viewing angle to the pole). For illustration, the results of two of these simulations are shown in Fig. 4. Here the profile of the fundamental feature at $\sim 10 \mathrm{keV}$ can be seen to vary considerably as a function of viewing angle. A detailed study of cyclotron line shapes with neutron star rotation has the potential to reveal a wealth of information about the conditions at the magnetic poles.

With the exception ${ }^{13}$ of a single observation of Her X-1 by the LEGS balloon-borne germanium spectrometer, however, no cyclotron absorption line in an accreting pulsar has ever been observed by an instrument with high enough resolution to distinguish among the many profiles shown in Fig. 4. Data from RHESSI and from the high-resolution SPI instrument on the INTEGRAL spacecraft to be launched this Fall will break new ground in this field. Coburn et al. ${ }^{10}$ point out that the systems with known cyclotron lines in their spectra are biased toward a specific viewing angle with respect to the magnetic field, and speculate that other pulsars might have narrower lines that remain undiscovered.

In addition, pulse-folding and high resolution will allow RHESSI to perform the most sensitive search to date for redshifted annihilation lines from the Crab and other pulsars. Of the many reports of such lines, the most challenging to test is a phase-dependent line seen at $440 \mathrm{keV}$ by the FIGARO II balloon instrument ${ }^{14}$ at $8 \times 10^{-5} \mathrm{ph} / \mathrm{cm}^{2} / \mathrm{s}$. RHESSI should be able to see the line at this flux using data already collected if the line is narrow.

Finally, the variation of pulse periods as a function of time is itself an important tool for studying accreting pulsars even without reference to spectroscopy. Different models of accretion flow onto a strongly magnetized 

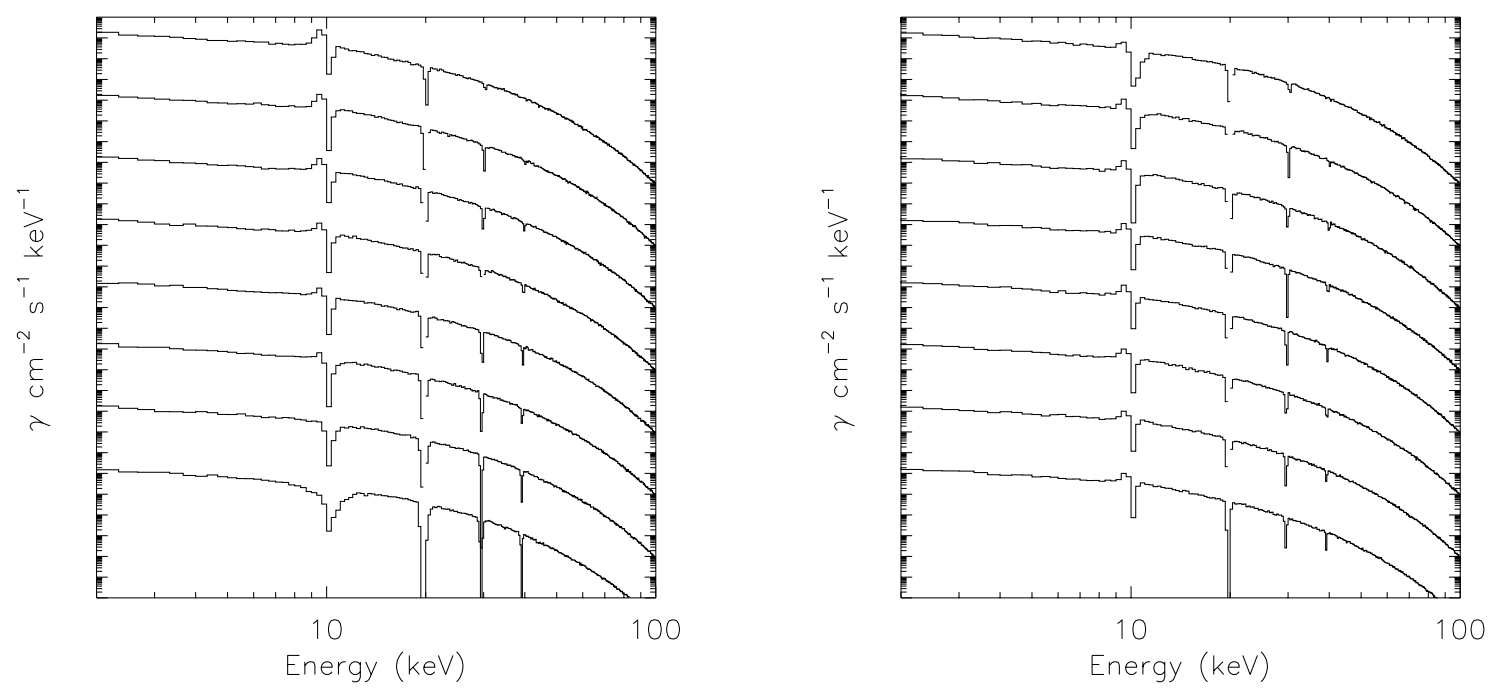

Figure 4. Monte-Carlo spectra from the isotropic injection of photons into two geometries. The dashed/histogram curves indicate the initial/scattered photon spectra. The 8 pairs of curves are for different viewing angles $(\theta)$ to the magnetic field, going from nearly perpendicular (bottom) to nearly parallel (top) and spaced evenly in $\cos \theta$. LEFT: Isotropic injection of photons into an idealized slab-plasma geometry. The cyclotron line fundamentals at low values of $\cos \theta$ are simple in shape and similar to observations, while at large $\cos \theta$ pronounced red wings are observed. RIGHT: Isotropic injection into an idealized cylindrical geometry. These lines are narrow, being virtually unresolved with the $1 / 3 \mathrm{keV}$ binning, and show minor red wings on the fundamental at all viewing angles.

neutron star have different predictions of spin-up and spin-down. Continuous observations of pulsars ${ }^{15}$ by the Burst and Transient Source Experiment (BATSE) on the Compton Gamma-Ray Observatory (CGRO) revealed unexpected complexities of spin behavior and frequent reversals of sign. This sort of observation has been unavailable since the termination of CGRO on June 4, 2000. Although RHESSI has a lower effective area than BATSE for these observations, there are still a number of known sources that are bright enough to follow. Furthermore, since every photon will be recovered with a time tag, RHESSI will not have the limitation of BATSE's normal operating mode, which returned rates every 2 seconds. We will be able to do a long-term survey of the poorly-sampled range of periods $<4 \mathrm{sec}$.

\section{OCCULTATION}

Another tremendous success of BATSE was its use in an Earth-occultation mode (EOM) to monitor hard xray sources continuously over the whole sky. ${ }^{17,18}$ Given the small mass to the sides of the Ge array (largely transparent above $\sim 30 \mathrm{keV}$ ), this becomes an attractive technique for RHESSI. Even though the RHESSI array has only about $20 \%$ of the geometric area of a single BATSE detector, the viability of this technique is dramatically illustrated in Fig. 5. This shows an occultation step from the bright black-hole transient Cygnus X-1 in RHESSI data collected on February 24, 2002, while this source was undergoing a very unusual bright outburst. ${ }^{19}$ The source observation time is shown compared with data taken exactly 15 orbits before and 15 orbits after it. There is a clear occultation edge when the source sets. Although very few sources will ever be bright enough to show such an obvious transition, combining data over many orbits at a time will allow several bright sources to be monitored. Estimating the source sensitivity by a simple scaling of the RHESSI background and effective area with respect to those of BATSE, we find a two-week sensitivity of $\sim 200 \mathrm{mCrab}$ and a daily sensitivity of $\sim 750 \mathrm{mCrab}$.

RHESSI's rotation, however, gives it a much more sensitive Detector Shadowing Mode ${ }^{20}$ (DSM) using detector-detector occultation for sources within about $25^{\circ}$ of the detector plane (i.e. nearly perpendicular to the Sun). An individual source will lie within this angular region for 4-6 months per year (split between two equal time periods), depending on distance from the ecliptic. Because there are roughly 10,000 detector/detector 


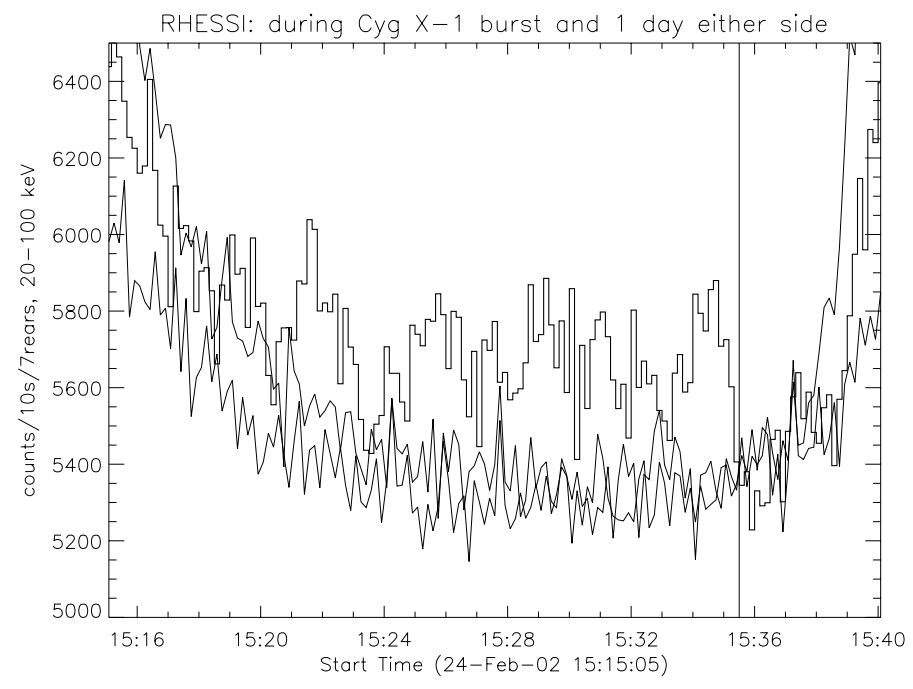

Figure 5. Occultation step of Cygnus X-1 on February 24, 2002. The histogram shows the data on February 24 during the outburst of the source. The other two traces are 15 orbits (about 1 day) before and after. The vertical line marks the time at which the source set (derived from the spacecraft ephemeris and the known source position, not the rate data themselves). At the beginning and end of the plot the spacecraft is in regions of higher background further from the Earth's magnetic equator.

occultations per day compared to only 15 or 16 Earth occultations, this technique has the potential to more than make up for the small size of the instrument compared to BATSE, as can be seen in Fig. 6 (left). Since the occultations are not abrupt or total, detailed modeling of the instrument response versus angle and energy will be necessary to implement this technique. Fig. 6 (right) shows the simulated response of one detector versus angle and energy. In a given energy band, the count rate versus time in each detector will be fit to known background components plus modulation profiles corresponding to the positions of known bright sources, with the possibility of adding "floating" sources to discover new bright transients.

\subsection{Occultation analysis of nuclear lines}

Another use of Earth occultation is the study of gamma-ray lines, both diffuse emission from the inner Galaxy and point sources. Gamma-ray line astrophysics as a field has been in suspension since the termination of CGRO, but RHESSI will have important, independent contributions to make during the renaissance anticipated with the launch of INTEGRAL.

The main technique for this analysis is an adaptation of an algorithm originally developed for doing astrophysics with the Solar Maximum Mission Gamma-Ray Spectrometer ${ }^{21}$ and later adapted for use with BATSE. ${ }^{22}$ In it simplest form, spectra taken when the source of interest was in eclipse are subtracted from spectra taken when it is visible. The underlying assumption is that nuclear lines are rare enough that there are not sources all over the sky for any given line. This is not, of course, strictly true, but for certain lines (such as diffuse lines from ${ }^{26} \mathrm{Al}$ and ${ }^{60} \mathrm{Fe}$ ) the inner Galaxy (within about $30^{\circ}$ Galactic longitude and $5^{\circ}$ Galactic latitude) is either known or expected to dominate.

Since the conditions that define the background may not on average be the same when the source is visible and when it is occulted, the algorithm is refined by selecting specific background pointings to match each source pointing based on the values of parameters that control background (cosmic-ray flux, spacecraft orientation with respect to the Earth, etc.). Spectra are accumulated over 1-minute intervals to form the database for this algorithm.

To improve the background subtraction even further, we can compensate for small residual differences in the background-controlling parameters between source and background. The background pointings, once selected, 

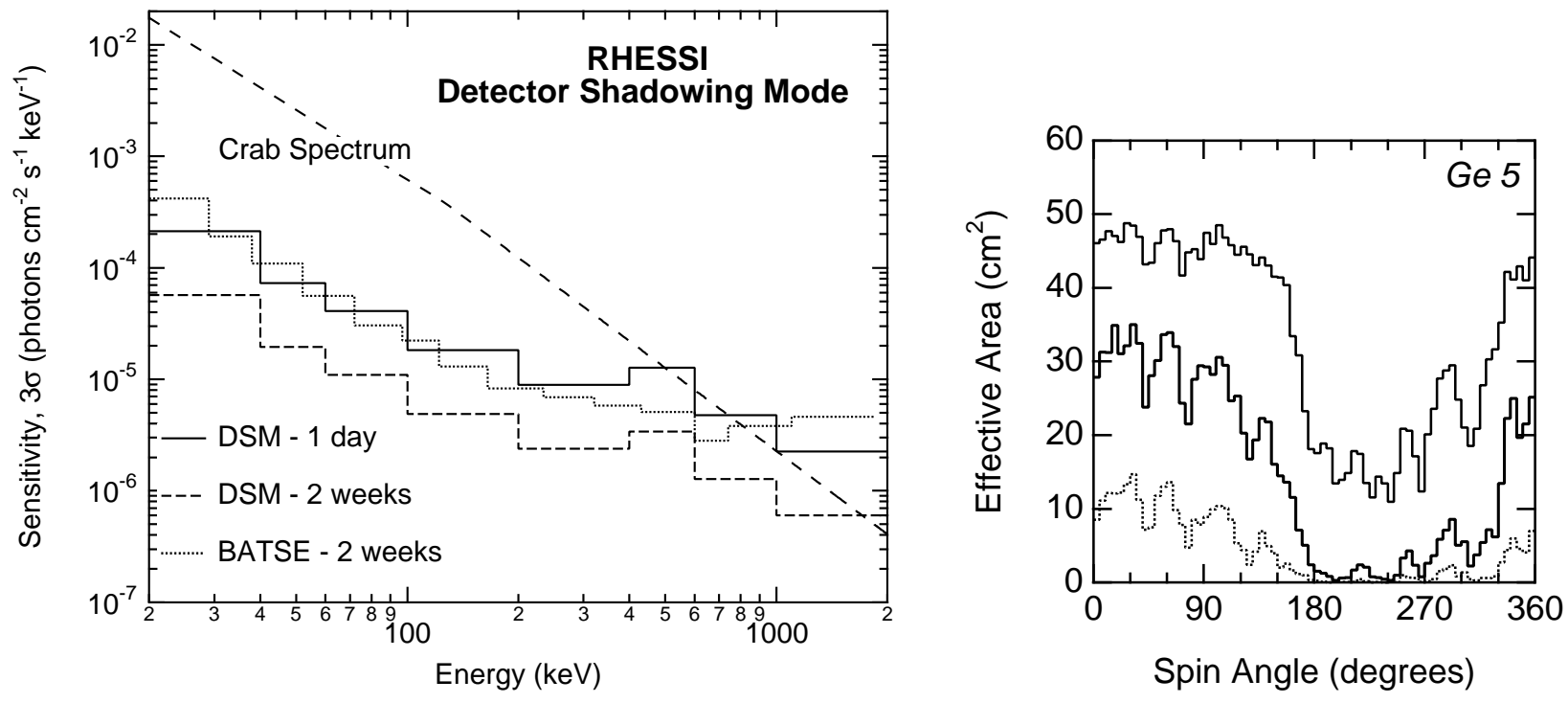

Figure 6. Left: continuum sensitivity for monitoring of sources using RHESSI in the Detector Shadowing Mode, with the BATSE EOM sensitivity and the spectrum ${ }^{16}$ of the Crab (nebula plus pulsar) for comparison. Right: Modulation pattern (effective area versus azimuth angle) expected for one of the RHESSI detectors for a cosmic source in the detector plane at $100 \mathrm{keV}, 50 \mathrm{keV}$, and $30 \mathrm{keV}$ (top to bottom).

are altered to match the source pointing more precisely by adding small multiples of "templates" that represent the average incremental change in background for an incremental change in each parameter. The templates are derived from comparisons among a large population of background pointings without reference to the source data. By this method, using four years of data and templates relating to eight different parameters, we derived the most precise measurement to date of the $511 \mathrm{keV}$ positron-annihilation line from the Galactic Center using BATSE. ${ }^{22}$

Important results awaiting confirmation by RHESSI using this method include:

1. The small (or zero) fraction of the Galactic $511 \mathrm{keV}$ flux that is in the relatively broad, $6.4 \mathrm{keV}$ FWHM component expected from annihilation in flight after charge exchange with neutral hydrogen. This result ${ }^{23}$ implies that Galactic positrons are mostly magnetically excluded from cold cloud cores.

2. The large width $\left(5.4(+1.4,-1.3) \mathrm{keV}\right.$ FWHM) measured for the integrated Galactic ${ }^{26} \mathrm{Al}$ line at 1809 $\mathrm{keV}$ line by the GRIS balloon instrument. ${ }^{24}$ This unexpectedly high width means that ${ }^{26} \mathrm{Al}$ ejected in supernovae maintains high velocities long after it would be expected to slow down in the ISM.

3. The low upper limit on the $1173 \mathrm{keV}$ line of ${ }^{60} \mathrm{Fe}$, also from GRIS, constraining models of supernova nucleosynthesis when compared to ${ }^{26} \mathrm{Al}$, under the assumption that most of the ${ }^{26} \mathrm{Al}$ is produced in supernovae.

A very preliminary analysis of 82 days of data from early in the mission seems to show the ${ }^{26} \mathrm{Al}$ line at about the expected flux and an upper limit (although not yet a scientifically meaningful one) on the ${ }^{60} \mathrm{Fe}$ line. None of the refinements of the background have yet been applied, however.

A modified version of this algorithm can be used when the source is known or suspected to be transient, lasting less than a day. If the background spectra are selected exactly 15 orbits (about 1 day) before and after each source spectrum, then the background and all the parameters that control it will be extremely close to their values at the time of the transient outburst (see Fig. 5 for the case of continuum emission sampled this way). Excellent background subtraction using only a few simple templates results. ${ }^{25}$ 
Our main transient-line target is positron-annihilation emission from classical novae. This is expected to appear in the first day of outburst, ${ }^{26,27}$ with most of the positrons produced by ${ }^{18} \mathrm{~F}$ and ${ }^{13} \mathrm{~N}$. Only upper limits have been observed to date, with the Transient Gamma-Ray Spectrometer (TGRS) on Wind ${ }^{28}$ and with BATSE. ${ }^{27}$ Because the line is blueshifted due to the velocity of the ejecta, high-resolution instruments like TGRS and RHESSI benefit from having the line offset from the strong annihilation background line. Our predicted $3 \sigma$ sensitivity for a 12-hour observation of a slightly blueshifted annihilation line is $8 \times 10^{-4} \mathrm{ph} / \mathrm{cm}^{2} / \mathrm{s}$, which is about a factor of three better than the existing limits. The existing limits are just about at the predicted flux levels ${ }^{27}$ so RHESSI gives us a good chance at the first real detection of annihilation in novae. INTEGRAL, although more sensitive, will not be able point to a nova in time: the annihilation radiation occurs before the optical detection, so only an all-sky instrument is likely to succeed.

\section{ACKNOWLEDGMENTS}

The authors would like to thank James Ling, Colleen Wilson-Hodge, Valerie Connaughton, William Wheaton, Manuel Guedel, Robert Lillis, James McTiernan, Martin Fivian, Gerald Share, and Margarida Hernanz for their contributions of ideas and their participation in RHESSI non-solar astronomy.

\section{REFERENCES}

1. C. Thompson and R. C. Duncan, "The soft gamma repeaters as very strongly magnetized neutron stars. II. Quiescent neutrino, x-ray, and Alfven wave emission," Ap. J. 473, pp. 322-342, 1996.

2. S. E. Woosley, "Gamma-ray bursts from stellar mass accretion disks around black holes," Ap. J. 405, pp. 273-277, 1993.

3. D. Eichler et al., "Nucleosynthesis, neutrino bursts and gamma-rays from coalescing neutron stars," Nature 340, pp. 126-128, 1989.

4. K. C. Hurley et al., "The ULYSSES supplement to the BATSE 3B catalog of cosmic gamma-ray bursts," Ap. J. Suppl. 120, pp. 399-408, 1999.

5. R. M. Pelling et al., "A scanning modulation collimator observation of the high-energy x-ray source in the Crab Nebula," Ap. J. 319, pp. 416-425, 1987.

6. J. J. Hester et al., "WFPC2 studies of the Crab Nebula. I. HST and ROSAT imaging of the synchrotron nebula," Ap. J. 448, pp. 240-263, 1995.

7. M. C. Weisskopf et al., "Discovery of spatial and spectral structure in the x-ray emission from the Crab Nebula," Ap. J. Letters 536, pp. L81-L84, 2000.

8. R. Willingale et al., "New light on the x-ray spectrum of the Crab Nebula," Ast. \& Ap. 365, pp. L212-217, 2001.

9. J. Trümper, W. Pietsch, C. Reppin, W. Voges, R. Staubert, and E. Kendziorra, "Evidence for strong cyclotron line emission in the hard X-ray spectrum of Hercules X-1," Ap. J. 219, p. L105, 1978.

10. W. Coburn, W. A. Heindl, R. E. Rothschild, D. E. Gruber, I. Kreykenbohm, J. Wilms, P. Kretschmar, and R. Staubert, 2002. Ap. J. in press.

11. R. Araya and A. Harding, "Cyclotron line features from near-critical magnetic fields: The effect of optical depth and plasma geometry," Ap. J. 517, p. 334, 1999.

12. R. Araya-Góchez and A. Harding, "Cyclotron line features from near-critical magnetic fields II: on the effect of anisotropic radiation fields," Ap. J. 544, 2000.

13. J. Tueller et al., "Evidence for variability of the hard X-ray feature in the Hercules X-1 energy spectrum," Ap. J. 279, pp. 177-183, 1984.

14. E. Massaro et al., "Detection of a feature at $0.44 \mathrm{MeV}$ in the Crab pulsar spectrum with FIGARO II - A redshifted positron annihilation line?," Ap. J. Letters 376, pp. L11-L15, 1991.

15. L. Bildsten et al., "Observations of accreting pulsars," Ap. J. Suppl. 113, pp. 367-408, 1997.

16. G. V. Jung, "The hard x-ray to low-energy gamma-ray spectrum of the Crab Nebula," Ap. J. 338, pp. 972982, 1989.

17. J. C. Ling et al., "A BATSE Earth occultation catalog of 0.03-1.8 MeV gamma-ray source spectra and light curves for phases 1-3 (1991-1994)," Ap. J. Suppl. 127, pp. 79-124, 2000. 
18. B. A. Harmon et al., "The BATSE Earth occultation catalog of low energy gamma-ray sources," Abstracts of the Joint American Physical Society/High Energy Astrophysics Division April Meeting, 2002. abstract \#B17.044.

19. S. Golenetskii et al., "Gamma-ray transient," IAU Circulars \#7840, 2002.

20. M. McConnell et al., 2002. in preparation.

21. M. Harris et al., "Measurement of the 0.3-8.5 MeV Galactic gamma-ray spectrum from the Galactic Center direction," Ap. J. 362, pp. 135-146, 1990.

22. D. M. Smith et al., "A BATSE measurement of the Galactic positron annihilation line," AIP Conf. Proceedings 410, pp. 1012-1016, 1997. Proc. of the 4th Compton Symposium.

23. M. Harris et al., "Transient Gamma-Ray Spectrometer measurements of the positron annihilation spectrum from the Galactic Center," Ap. J. Lett. 501, pp. L55-L58, 1998.

24. J. Naya et al., "Detection of high-velocity ${ }^{26} \mathrm{Al}$ towards the Galactic Centre," Nature 384, p. 44, 1996.

25. D. M. Smith et al., "All-sky search for transient souces near 0.5 Mev with the Burst and Transient Source Experiment (BATSE)," Ap. J. 471, pp. 783-795, 1996.

26. J. Gomez-Gomar et al., "Gamma-ray emission from individual classical novae," MNRAS 296, pp. 913-920, 1998.

27. M. Hernanz et al., "BATSE observations of classical novae," AIP Conf. Proceedings 510, p. 82, 2000. Proc. of the 5th Compton Symposium.

28. M. Harris et al., "Transient Gamma Ray Spectrometer observations of gamma-ray lines from novae. I. Limits on the positron annihilation line in five individual novae," Ap. J. 522, pp. 424-432, 1999. 